\section{Optical Imaging: Skin Cancer Imaging}

TO THE EDITOR: The excellent review article by Luker and Luker (1) on the current and future directions is well done and presents most of the emerging optical imaging modalities-except skin cancer imaging.

Skin cancer is often the stepchild of cancer research and typically does not get the recognition it may deserve. Yet skin malignancies represent the highest number of new cancers reported every year, at over 1 million annually, based on estimations by the American Cancer Society (2). This number represents approximately $40 \%$ of all new cancers detected in the United States per year. However, most of these cancers are excluded from cancer statistics published by the American Cancer Society. Therefore, skin cancers, such as basal cell carcinoma and squamous cell carcinoma, are often ignored or overlooked in discussions of cancer research.

Skin cancer detection is one of the best applications of optical imaging. With recent imaging advancements, optical imaging for skin cancer diagnosis has become an indispensible clinical tool that is used by $22 \%$ of dermatologists in the United States (3). Indeed, molecular optical imaging of skin cancer is routinely used clinically for imaging fluorescence from basal cell carcinomas using $\delta$-5-aminolaevulinic acid as a fluorescent probe (4). More recently, molecular optical imaging studies with a fluorescent analog of deoxyglucose (2-( $N$-(7-nitrobenz-2-oxa-1,3-diazol-4yl)amino)-2-deoxyglucose) have demonstrated that such probes hold the promise of providing an optical analog of FDG for metabolism mapping of superficial skin cancer (5).

Skin cancer is a powerful application of optical imaging in medicine. Because most skin cancers are located superficially, applying optical imaging methods is not as daunting a problem as imaging deeper cancers, such as breast cancer. Further, optical imaging equipment does not have to be expensive to be medically useful. Devices such as the dermatoscope (6) and DermLite (3Gen, LLC) (7) are inexpensive and, in the case of DermLite devices, can be adapted to provide multispectral and fluorescence imaging capabilities. So, when reviewing optical imaging methods in medicine, one must also consider the important clinical role they play in the diagnosis of skin cancer and treatment follow-up. Unlike most of the applications described by Luker and Luker (1), optical imaging of skin cancer is already being used clinically and could help pave the way for molecular optical imaging in medicine.

\section{REFERENCES}

1. Luker GD, Luker KE. Optical imaging: current applications and future directions. J Nucl Med. 2008;49:1-4.

2. American Cancer Society. Cancer Facts \& Figures 2007. Atlanta, GA: American Cancer Society; 2007.

3. Federman DG, Kravetz JD, Kirsner RS. Skin cancer screening by dermatologists: prevalence and barriers. J Am Acad Dermatol. 2002;46:710-714.

4. Szeimies RM, Landthaler M. Photodynamic therapy and fluorescence diagnosis of skin cancers. Recent Results Cancer Res. 2002;160:240-245.

COPYRIGHT @ 2008 by the Society of Nuclear Medicine, Inc.
5. O'Neil RG, Wu L, Mullani N. Uptake of fluorescent analog (2-NBDG) in tumor cells. Mol Imaging Biol. 2005;7:388-392.

6. Braun-Falco O, Stolz W, Bilek P, Merkle T, Landthaler M. The dermatoscope: a simplification of epiluminescent microscopy of pigmented skin changes [in German]. Hautarzt. 1990;41:131-136.

7. Arrazola P, Mullani NA, Abramovits W. DermLite II: an innovative portable instrument for dermoscopy without the need for immersion fluids. Skinmed. 2005;4: 78-83.

Nizar A. Mullani 3GEN LLC

San Juan Capistrano, California

TransLite LLC

Sugar Land, Texas

Roger G. O'Neil

University of Texas Medical School

Houston, Texas

DOI: 10.2967/jnumed.108.051185

\section{Feasibility of Automated Partial-Volume Correction of SUVs in Current PET/CT Scanners: Can Manufacturers Provide Integrated, Ready-to- Use Software?}

TO THE EDITOR: The article by Soret et al. (1) in the June 2007 issue of The Journal of Nuclear Medicine on the implications of partial-volume effect (PVE) correction for PET in cancer provides a timely review of this important subject. It is quite clear that educating physicians-who are actively involved in the interpretation of PET images-is essential to correct for errors attributable to PVE. We congratulate the authors for their scholarly scientific communication and share our views on this topic in quantitative PET.

In recent years, a number of reports (1-3) have raised concerns about errors introduced by PVE in the quantitative assessment of tracer concentration with PET at intended sites. Although the initial reports dealt primarily with the measurement of metabolic values in the brain in different neurologic disorders (4-7), this effect has been increasingly recognized recently in malignant lesions $(1-3,8-10)$. In addition to its importance in diagnosis, PVE correction has serious implications for treatment monitoring, an area in which a change in the standardized uptake value (SUV) is often used as an objective parameter to assess treatment response and in which lesions frequently become smaller after successful therapy. It is now proven that adoption of even a simple correction approach such as the recovery coefficient method can substantially reduce the error in uptake estimates of the lesions being examined.

The routine implementation of PVE correction in clinical practice is technically demanding and is an obstacle in most settings. By now, multiple clinical validation studies have addressed the 
importance of PVE correction and have suggested its use for accurate assessment of disease activity. However, successful implementation of this procedure would require a reproducible and relatively simple approach that can be performed on all patients.

Several PVE correction methods, including the popular recovery coefficient technique, require measuring the lesion dimensions in anatomic images such those produced by CT or MRI. The size estimate from current PET/CT scanners provides the opportunity to determine the PVE-corrected SUV readily. We believe the everincreasing use of cross-sectional anatomic imaging with either CT or MRI along with the current-generation PET scanners can make this goal achievable through automated algorithms that provide the PVE-corrected SUV directly and easily. Until recently, the widespread use of PVE correction has been hampered primarily by the lack of integrated processing software with these hybrid scanners. With the use of fusion imaging in clinical practice becoming widespread, these limitations likely will not persist. We believe that manufacturers can assess the recovery coefficients for PVE correction for a particular scanner and integrate them into these scanners before they are installed at the site. The availability of a PVE-corrected SUV will be a strong step toward the routine use of this procedure at the clinical level, ultimately improving patient care. We speculate that eventually most scanners will have integrated automated PVE correction software. Both practitioners and the industry should be aware of the potential advantages of this evolution and take active steps toward bringing it about. Compared with the current schemes, integrated PVE software will strengthen the role and reliability of the SUV measurement as a quantitative measure.

In addition to the impact on routine patient care, such correction methods are expected to enhance the research applications of PET in the field of clinical oncology and other related disciplines. With PET/CT facilities being established in many centers across the world, it would be desirable for accurate clinical data to be generated not only from advanced and large university centers but also from small centers in communities with varying levels of expertise. The availability of integrated algorithms would likely improve reproducibility and reduce operator-dependent errors. Adoption of automated correction procedures that have been integrated with existing software will truly revolutionize the impact of this powerful modality worldwide. Every effort should be made toward achieving this goal.

\section{ACKNOWLEDGMENT}

This work was supported in part by the International Union against Cancer (UICC), Geneva, Switzerland, under the ACSBI fellowship.

\section{REFERENCES}

1. Soret M, Bacharach SL, Buvat I. Partial-volume effect in PET tumor imaging. J Nucl Med. 2007;48:932-945.

2. Basu S, Alavi A. Partial volume correction of standardized uptake values and the dual time point in FDG-PET imaging: should these be routinely employed in assessing patients with cancer? Eur J Nucl Med Mol Imaging. 2007;34:1527-1529.

3. Basu S, Zaidi H, Houseni M, et al. Novel quantitative techniques for assessing regional and global function and structure based on modern imaging modalities: implications for normal variation, aging and diseased states. Semin Nucl Med. 2007;37:223-239.

4. Meltzer CC, Leal JP, Mayberg HS, Wagner HN Jr, Frost JJ. Correction of PET data for partial volume effects in human cerebral cortex by MR imaging. J Comput Assist Tomogr. 1990;14:561-570.
5. Chawluk JB, Alavi A, Dann R, et al. Positron emission tomography in aging and dementia: effect of cerebral atrophy. J Nucl Med. 1987;28:431-437.

6. Tanna NK, Kohn MI, Horwich DN, et al. Analysis of brain and cerebrospinal fluid volumes with MR imaging: impact on PET data correction for atrophy. Part II. Aging and Alzheimer dementia. Radiology. 1991;178:123-130.

7. Alavi A, Newberg AB, Souder E, Berlin JE. Quantitative analysis of PET and MRI data in normal aging and Alzheimer's disease: atrophy weighted total brain metabolism and absolute whole brain metabolism as reliable discriminators. J Nucl Med. 1993;34:1681-1687.

8. Hickeson M, Yun M, Matthies A, et al. Use of a corrected standardized uptake value based on the lesion size on CT permits accurate characterization of lung nodules on FDG-PET. Eur J Nucl Med Mol Imaging. 2002;29:1639_ 1647 .

9. Alkhawaldeh K, Bural G, Kumar R, Alavi A. Impact of dual-time-point ${ }^{18} \mathrm{~F}-\mathrm{FDG}$ PET imaging and partial volume correction in the assessment of solitary pulmonary nodules. Eur J Nucl Med Mol Imaging. 2007 October 16 [Epub ahead of print].

10. Avril N, Rosé CA, Schelling M, et al. Breast imaging with positron emission tomography and fluorine-18 fluorodeoxyglucose: use and limitations. J Clin Oncol. 2000;18:3495-3502.

\author{
Sandip Basu \\ Hospital of University of Pennsylvania \\ Philadelphia, Pennsylvania \\ Radiation Medicine Center (BARC) \\ Tata Memorial Hospital Annexe \\ Parel, Bombay, India
}

\author{
Abass Alavi \\ Hospital of University of Pennsylvania \\ Philadelphia, Pennsylvania
}

DOI: 10.2967/jnumed.108.050401

REPLY: We appreciate our colleagues' observations about corrections for the partial-volume effect (PVE) in PET. They have brought up an important and timely issue-whether PET/CT manufacturers could offer PVE corrections as part of their clinical software. We agree that even if no perfectly accurate PVE correction has been designed so far, many possibilities do exist to reduce the bias introduced by PVE without substantially increasing variability (1). As mentioned in our review paper, by showing the anatomic support of functional abnormalities, fused PET/CT images (and soon PET/MR images) make it possible to implement simple PVE corrections, such as those based on recovery coefficients $(2,3)$. Simpler corrections that do not even require any assumption regarding the contours of the functional abnormalities (4) are also available. Even if such corrections remain approximate, having them available could provide the user with an extremely valuable tool to assess the reliability of the SUV estimates, permitting computation of the SUV both with and without PVE correction, similar to showing both attenuation-corrected and non-attenuation-corrected images.

Having both the corrected and uncorrected SUV indices available to the physicians would seem to be a great step forward toward a sounder quantitative interpretation of the images. This is true even if the corrections might be only approximate. As mentioned in the letter of Drs. Basu and Alavi, this is especially true in the context of patient monitoring, an area in which changes 